\title{
Two-stage decoding algorithm for unmodulated parallel combinatory high-compaction multicarrier modulation signals
}

\author{
Ryuji Hayashi, Yingsong Li ${ }^{*}$ and Masanori Hamamura
}

\begin{abstract}
A new decoding algorithm that consists of two decoding stages to reduce the computational complexity of maximum likelihood decoding for parallel combinatory high-compaction multicarrier modulation is proposed. The first decoding stage is responsible for a preliminary decision that serves to roughly find candidate messages using the QRD-M algorithm, and the second decoding stage is responsible for the final decision that reduces the error contained in the candidate and determines the message using the minimum value of the Hamming distances between the candidate and the replicas of the message. The complexity is considerably reduced by the proposed two-stage decoding algorithm at a cost of approximately $1.5 \mathrm{~dB}$ or less in $E_{b} / N_{0}$ with respect to the bit-error rate of $10^{-3}$ under the given parameter settings.
\end{abstract}

Keywords: PC signaling, HC-MCM, QRD-M algorithm, Maximum likelihood decoding

\section{Introduction}

Recently, multicarrier transmission systems have been investigated from the viewpoint of high-speed communication and high spectral efficiency for wireless communication. Orthogonal frequency-division multiplexing (OFDM) is a well-known multicarrier transmission system that has a high transmission rate and high resistance to multipath interference over the multipath channel [1-3]. On the other hand, parallel combinatory (PC) signaling methods have been investigated as methods for improving spectral efficiency [4-8]. Parallel combinatory OFDM (PC-OFDM) was proposed in a previous article [9] and uses the technique of PC signaling, which has higher spectral efficiency and a lower bit-error rate (BER) than ordinary OFDM. Another technique that improves the spectral efficiency of OFDM is high-compaction multicarrier modulation (HC-MCM) [10]. This is a method of achieving high spectral efficiency by overlapping the carriers of the transmitted signal. Parallel combinatory

*Correspondence: $156011 y @ g s . k o c h i-t e c h . a c . j p$

Graduate School of Engineering, Kochi University of Technology, Kami-shi 782-8502, Japan
HC-MCM (PC/HC-MCM) [11] was proposed using the techniques of $\mathrm{PC}$ signaling and HC-MCM. Parallel combinatory $\mathrm{HC}-\mathrm{MCM}$ can be categorized as either modulated $\mathrm{PC} / \mathrm{HC}-\mathrm{MCM}$ or unmodulated PC/HC-MCM. Modulated $\mathrm{PC} / \mathrm{HC}-\mathrm{MCM}$ transmits a truncated PC-OFDM signal, which is a PC-OFDM signal multiplied by a window function in the time domain, and improves the BER performance of HC-MCM. Unmodulated PC/HC-MCM transmits a truncated PC-OFDM signal without $N$-ary amplitude phase shift keying (APSK) modulation and improves the peak-to-average power ratio (PAR) of HCMCM. In both types of PC/HC-MCM, the transmission rate can be chosen flexibly by controlling the window width, which changes the modulation index of $\mathrm{PC} / \mathrm{HC}$ MCM. Therefore, PC/HC-MCM can be designed to have a lower PAR and higher spectrum efficiency than conventional OFDM and is suitable for consumer electronic device applications, such as sensor networks and wireless home networks. PC/HC-MCM is also compatible with the conventional OFDM used in wireless multimedia, digital audio/video broadcasting (DAB/DVB), and so forth.

Maximum likelihood (ML) decoding [12] has been considered as a means of recovering message data with 
a lower BER in PC/HC-MCM. However, the computational complexity for demodulation using ML decoding increases with the number of combinations of PC signaling. An effective method that reduces the computational complexity for ML decoding is to reduce the number of Euclidean distance calculations required to identify the transmitted message data using the $(M)$-algorithm at the receiver [13,14].

In this article, a method using the QRD-M algorithm $[15,16]$, which uses QR decomposition (QRD) and the $(M)$-algorithm [17] to improve the demodulation efficiency of PC/HC-MCM, is proposed and its performance is verified over an additive white Gaussian noise (AWGN) channel. This algorithm consists of two decoding stages. The first decoding stage is responsible for a preliminary decision that serves to roughly find candidate message data using the QRD-M algorithm. The second decoding stage is responsible for a final decision that corrects the error contained in the candidate decoded at the first decoding stage. Although another type of two-stage decoding algorithm has been considered [18], we have modified it to more efficiently and reliably decode the message data.

The remainder of this article is organized as follows. In Section 2, PC-OFDM and unmodulated PC/HCMCM are introduced and ML decoding is formulated. In Section 3, the proposed decoding algorithm is described. In Section 4, the computational complexity and BER performance of $\mathrm{PC} / \mathrm{HC}-\mathrm{MCM}$ using the proposed decoding algorithm are discussed. Finally, this article is concluded in Section 5.

\section{Unmodulated PC/HC-MCM}

\subsection{PC-OFDM}

PC-OFDM is a type of OFDM that conveys message data with PC signaling together with ordinary $\mathrm{N}$-ary amplitude and phase shift keying ( $N$-ary APSK).

Let $M_{c}$ be the total number of preassigned carriers and $M_{p}$ be the number of carriers selected for PC signaling. In this case, the number of message data bits per PC-OFDM signal, $m_{\text {total }}$ [bits], is represented as

$$
m_{\mathrm{total}}=m_{\mathrm{apsk}}+m_{p c}
$$

where $m_{\text {apsk }}$ [bits] is the number of message data bits mapped into $N$-ary APSK constellations of $M_{p}$ carriers, given by

$$
m_{\text {apsk }}=M_{p} \log _{2} N
$$

and $m_{p c}$ [bits] is the number of message data bits encoded into one of the prescribed sets of $M_{p}$ carriers, that is,

$$
m_{p c}=\left\lfloor\log _{2}\left(\begin{array}{l}
M_{c} \\
M_{p}
\end{array}\right)\right\rfloor,
$$

where $\lfloor\cdot\rfloor$ is an operator that denotes the largest integer that is smaller than or equal to the operand.
The PC-OFDM signals are modeled as

$$
y(t)=\sum_{l=1}^{M_{c}} x_{l} e^{j 2 \pi(l-1) \Delta f t}, \quad 0 \leqq t<T_{0},
$$

where $x_{l}\left(l=1,2, \ldots, M_{c}\right)$ is the complex symbol for the $l$ th carrier, which takes an $(N+1)$-ary APSK constellation including zero amplitude; in $0 \leqq t<T_{0}, T_{0}=1 / \Delta f$ [s] is the duration of one PC-OFDM signal and $\Delta f[\mathrm{~Hz}]$ is the frequency spacing.

Here, $m_{p c}$ message data bits can be transmitted without $N$-ary APSK in PC-OFDM. In this case, $x_{l} \in\{0,1\}$ and $m_{\text {total }}=m_{p c}$.

\section{$2.2 \mathrm{PC} / \mathrm{HC}-\mathrm{MCM}$}

Similarly to PC-OFDM, PC/HC-MCM is a multicarrier modulation scheme that uses $\mathrm{PC}$ signaling. $\mathrm{PC} / \mathrm{HC}-\mathrm{MCM}$ can be categorized as either modulated PC/HC-MCM or unmodulated PC/HC-MCM. Modulated PC/HC-MCM transmits a truncated PC-OFDM signal, and unmodulated PC/HC-MCM transmits a truncated PC-OFDM signal without $N$-ary APSK modulation. In this article, we consider unmodulated PC/HC-MCM to take advantage of one of its favorable properties. For example, we can choose $\left(\begin{array}{l}M_{c} \\ M_{p}\end{array}\right)=\left(\begin{array}{c}16 \\ 8\end{array}\right)$ and modulation index 0.5 to transmit 13 bits message data. This means that the unmodulated $\mathrm{PC} / \mathrm{HC}-\mathrm{MCM}$ system can transmit 13 bits data within a time duration of $0.5 T_{0}$ using $16 \Delta f$ bandwidth. In other words, the unmodulated $\mathrm{PC} / \mathrm{HC}-\mathrm{MCM}$ system can transmit $2 \times 13=26$ bits within $1 T_{0}$ using $16 \Delta f$ bandwidth, which indicates that the spectral efficiency of the unmodulated PC/HC-MCM system is better than that of the OFDM with BPSK which can transmit only 16 bits within $1 T_{0}$ using $16 \Delta f$ bandwidth. We hereinafter refer to unmodulated $\mathrm{PC} / \mathrm{HC}-\mathrm{MCM}$ simply as PC/HC-MCM.

Let $\mathcal{A}=\left\{k \in \mathbb{N} \mid 1 \leqq k \leqq M_{c}\right\}$ be the set of frequency indices of all preassigned carriers, and let $\mathcal{B}=$ $\left\{b_{1}, b_{2}, \ldots, b_{M_{p}}\right\} \subset \mathcal{A}\left(|\mathcal{B}|=M_{p}\right.$, where $|\cdot|$ is the cardinality, that is, the number of elements in the set) be a subset of $\mathcal{A}$, which is the set of the frequency indices of carriers used for PC signaling. Without loss of generality, we assume that $b_{1}<b_{2}<\cdots<b_{M_{p}}$. Note that $\mathcal{B}$ is a possible subset of $\mathcal{A}$, that is, $\mathcal{B} \in \mathcal{C}=\left\{\mathcal{B}_{1}, \mathcal{B}_{2}, \ldots, \mathcal{B}_{2}{ }^{m p c}\right\}$. In this case, the $\mathrm{PC} / \mathrm{HC}-\mathrm{MCM}$ signal can be written in a complex low-pass equivalent notation as

$$
y(t)=w(t) \sum_{l \in \mathcal{B}} e^{j 2 \pi(l-1) \Delta t}, \quad 0 \leqq t<T_{s},
$$

where $T_{s}$ is the duration $[\mathrm{s}]$ and is chosen to be $T_{s}<1 / \Delta f$ for $\mathrm{PC} / \mathrm{HC}-\mathrm{MCM}$, and $w(t)$ is a window function defined in $0 \leq t<T_{s}$. In this article, $w(t)$ is assumed to be $w(t)=$ $\sin \left(\frac{\pi}{T_{s}} t\right)$, which is a finite-duration window that minimizes the root mean square (RMS) bandwidth of each carrier that is transmitted [19]. 


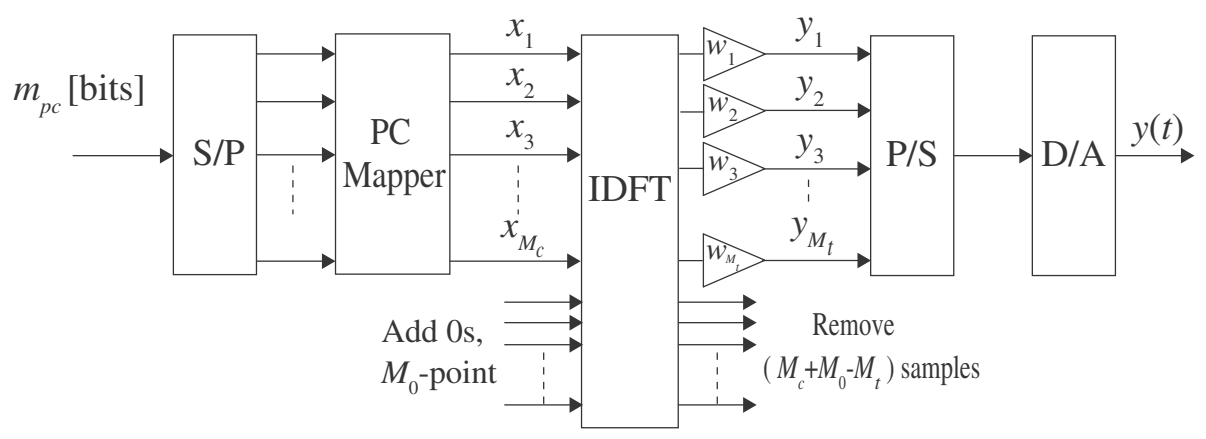

Figure 1 IDFT-based transmitter of PC/HC-MCM.

The above expression can be rewritten as

$$
y(t)=w(t) \sum_{l=1}^{M_{c}} x_{l} e^{j 2 \pi(l-1) \Delta f t}, 0 \leqq t<T_{s},
$$

where

$$
x_{l}=\left\{\begin{array}{ll}
1 & (l \in \mathcal{B}) \\
0 & (l \notin \mathcal{B})
\end{array} .\right.
$$

Therefore, an inverse discrete Fourier transform (IDFT) transmitter for PC/HC-MCM can be configured using (6) as shown in Figure 1 . At the transmitter, $m_{p c}$ message data bits are subjected to serial-to-parallel (S/P) conversion and are mapped into one of the possible $2^{m_{p c}}$ combinations of $M_{p}$ carriers, the frequencies of which are characterized by the set $\mathcal{B}$ of frequency indices. An example of PC mapping using the Gray code for $\left(\begin{array}{l}M_{c} \\ M_{p}\end{array}\right)=\left(\begin{array}{c}8 \\ 2\end{array}\right)$ is given in Table 1.

At the transmitter of PC/HC-MCM, $M_{0}$ zeros are tacked onto the binary sequence $x_{l}\left(l=1,2, \ldots, M_{c}\right)$ as padding at the input of the IDFT for the interpolation of discrete-time samples obtained by the IDFT. After the IDFT, we obtain $M_{c}+M_{0}$ samples and remove $\left(M_{c}+\right.$ $\left.M_{0}-M_{t}\right)$ samples. Therefore, only $M_{t}$ samples, weighted by gain constants $w_{m}=\sin \left(\frac{m-1}{M_{t}} \pi\right)\left(m=1,2, \ldots, M_{t}\right)$, are used for parallel-to-serial $(\mathrm{P} / \mathrm{S})$ conversion and digitalto-analog (D/A) conversion to produce the transmitting signal $y(t)$.

At the receiver shown in Figure 2, the signal $y(t)$ is detected in the presence of an AWGN $n(t)$ with a doublesided power spectral density of $N_{0} / 2$. After the A/D converter, we obtain the discrete-time samples $\hat{y}_{m} \quad(m=$ $\left.1,2, \ldots, M_{t}\right)$ detected at time $t=(m-1) T_{s} / M_{t}$, that is,

$$
\hat{y}_{m}=(y(t)+\eta(t))_{t=(m-1) T_{s} / M_{t}},
$$

where $\eta(t)$ is the noise obtained at the A/D converter output.

Let $\hat{\mathbf{y}}$ be a column vector that contains the samples $\hat{y}_{m}$ such that

$$
\hat{\mathbf{y}}=\left[\begin{array}{llll}
\hat{y}_{1} & \hat{y}_{2} & \ldots & \hat{y}_{M_{t}}
\end{array}\right]^{T},
$$

and let $\mathbf{x}_{\mathcal{B}}=\left[\begin{array}{llll}x_{1} & x_{2} & \ldots & x_{M_{c}}\end{array}\right]^{T}$ be a column vector that has the elements $x_{l}$ defined as (7). The IDFT transmitter is expressed by the submatrix $\mathbf{F}$ of the full IDFT matrix, given by

$$
\mathbf{F}=\left[\begin{array}{ccc}
\omega_{1,1} & \cdots & \omega_{1, M_{c}} \\
\vdots & \ddots & \vdots \\
\omega_{M_{t}, 1} & \cdots & \omega_{M_{t}, M_{c}}
\end{array}\right]
$$

where

$$
\omega_{m, l}=\frac{1}{\sqrt{M_{c}+M_{0}}} e^{j \frac{2 \pi(l-1)(m-1)}{M_{c}+M_{0}}} .
$$

\begin{tabular}{|c|c|c|c|}
\hline Message data & $\mathcal{B}_{i}$ & Frequencies & $\mathrm{x}_{\mathcal{B}_{i}}=\left[x_{i, 1} x_{i, 2} \ldots x_{i, 8}\right]^{T}$ \\
\hline 1100 & $\{1,2\}$ & $\left\{f_{1}, f_{2}\right\}$ & {$\left[\begin{array}{llllllll}1 & 1 & 0 & 0 & 0 & 0 & 0 & 0\end{array}\right]^{\top}$} \\
\hline 1101 & $\{1,3\}$ & $\left\{f_{1}, f_{3}\right\}$ & {$\left[\begin{array}{llllllll}1 & 0 & 1 & 0 & 0 & 0 & 0 & 0\end{array}\right]^{T}$} \\
\hline 1111 & $\{1,4\}$ & $\left\{f_{1}, f_{4}\right\}$ & {$\left[\begin{array}{llllllll}1 & 0 & 0 & 1 & 0 & 0 & 0 & 0\end{array}\right]^{\top}$} \\
\hline 1110 & $\{1,5\}$ & $\left\{f_{1}, f_{5}\right\}$ & {$\left[\begin{array}{llllllll}1 & 0 & 0 & 0 & 1 & 0 & 0 & 0\end{array}\right]^{T}$} \\
\hline 1010 & $\{1,6\}$ & $\left\{f_{1}, f_{6}\right\}$ & {$\left[\begin{array}{llllllll}1 & 0 & 0 & 0 & 0 & 1 & 0 & 0\end{array}\right]^{\top}$} \\
\hline 1011 & $\{1,7\}$ & $\left\{f_{1}, f_{7}\right\}$ & {$\left[\begin{array}{lllllllll}1 & 0 & 0 & 0 & 0 & 0 & 1 & 0\end{array}\right]^{T}$} \\
\hline 1001 & $\{1,8\}$ & $\left\{f_{1}, f_{8}\right\}$ & {$\left[\begin{array}{llllllll}1 & 0 & 0 & 0 & 0 & 0 & 0 & 1\end{array}\right]^{\top}$} \\
\hline 1000 & $\{2,3\}$ & $\left\{f_{2}, f_{3}\right\}$ & {$\left[\begin{array}{llllllll}0 & 1 & 1 & 0 & 0 & 0 & 0 & 0\end{array}\right]^{\top}$} \\
\hline 0000 & $\{2,4\}$ & $\left\{f_{2}, f_{4}\right\}$ & {$\left[\begin{array}{llllllll}0 & 1 & 0 & 1 & 0 & 0 & 0 & 0\end{array}\right]^{\top}$} \\
\hline 0001 & $\{2,5\}$ & $\left\{f_{2}, f_{5}\right\}$ & {$\left[\begin{array}{llllllll}0 & 1 & 0 & 0 & 1 & 0 & 0 & 0\end{array}\right]^{T}$} \\
\hline 0011 & $\{2,6\}$ & $\left\{f_{2}, f_{6}\right\}$ & {$\left[\begin{array}{llllllll}0 & 1 & 0 & 0 & 0 & 1 & 0 & 0\end{array}\right]^{\top}$} \\
\hline 0010 & $\{2,7\}$ & $\left\{f_{2}, f_{7}\right\}$ & {$\left[\begin{array}{llllllll}0 & 1 & 0 & 0 & 0 & 0 & 1 & 0\end{array}\right]^{\top}$} \\
\hline 0110 & $\{2,8\}$ & $\left\{f_{2}, f_{8}\right\}$ & {$\left[\begin{array}{llllllll}0 & 1 & 0 & 0 & 0 & 0 & 0 & 1\end{array}\right]^{T}$} \\
\hline 0111 & $\{3,4\}$ & $\left\{f_{3}, f_{4}\right\}$ & {$\left[\begin{array}{llllllll}0 & 0 & 1 & 1 & 0 & 0 & 0 & 0\end{array}\right]^{\top}$} \\
\hline 0101 & $\{3,5\}$ & $\left\{f_{3}, f_{5}\right\}$ & {$\left[\begin{array}{llllllll}0 & 0 & 1 & 0 & 1 & 0 & 0 & 0\end{array}\right]^{T}$} \\
\hline 0100 & $\{3,6\}$ & $\left\{f_{3}, f_{6}\right\}$ & {$\left[\begin{array}{llllllll}0 & 0 & 1 & 0 & 0 & 1 & 0 & 0\end{array}\right]^{\top}$} \\
\hline
\end{tabular}

Therefore, $\hat{\mathbf{y}}$ is expressed using $\mathbf{F}$ and $\mathbf{x}_{\mathcal{B}}$ as

$$
\hat{\mathbf{y}}=\mathbf{W F x _ { \mathcal { B } } + \eta},
$$




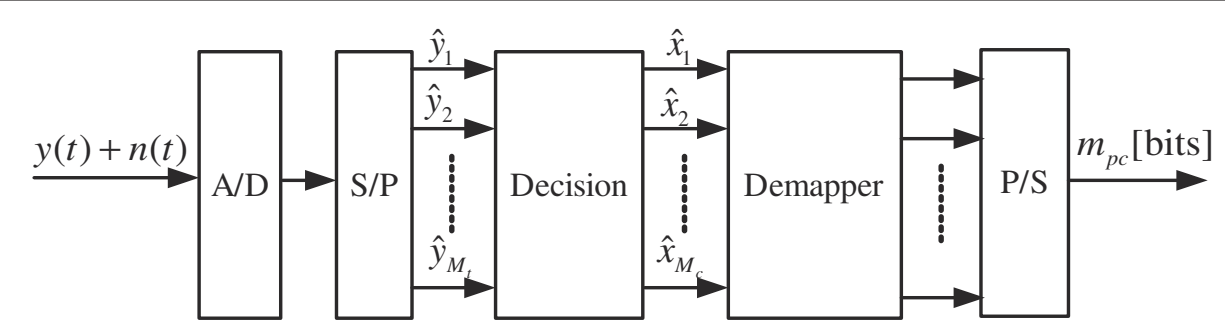

Figure 2 Receiver of PC/HC-MCM.

where $\eta$ is a column vector that contains the samples of the noise term $\eta(t)$ and $\mathbf{W}$ is a diagonal matrix that has the weights $w_{m}\left(m=1,2, \ldots, M_{t}\right)$ in its main diagonal, that is,

$$
\mathbf{W}=\operatorname{diag}\left\{w_{1}, w_{2}, \ldots, w_{M_{t}}\right\}
$$

\subsection{Maximum likelihood decoding}

The decision stage determines which PC/HC-MCM signal, characterized by the set $\mathcal{B}$ of indices of carriers, was transmitted. In this problem, from all possible sets of $\mathcal{B}_{i} \in$ $\mathcal{C}$, ML decoding finds the set $\hat{\mathcal{B}}$ that satisfies the following formula:

$$
\hat{\mathcal{B}}=\arg \min _{\mathcal{B}_{i} \in \mathcal{C}}\left(J\left(\mathcal{B}_{i}\right)=\left\|\hat{\mathbf{y}}-\mathbf{W F} \mathbf{x}_{\mathcal{B}_{i}}\right\|\right)
$$

where $\mathbf{W} \mathbf{F} \mathbf{x}_{\mathcal{B}_{i}}\left(i=1,2, \ldots, 2^{m p c}\right)$ are the replica vectors, that is, the noiseless version of vector $\hat{\mathbf{y}}$, and $\|\cdot\|$ denotes the Euclidean norm of the enclosed vector.

Maximum likelihood decoding is an optimization method that minimizes the probability of an error in finding the set $\mathcal{B}$ used to transmit the PC/HC-MCM signal. However, since the value of $2^{m_{p c}}$ is a very large integer when $\left(\begin{array}{l}M_{c} \\ M_{p}\end{array}\right)$ is large, it is difficult to solve such a problem for $M_{c} \gg 1$ and $M_{p} \approx M_{c} / 2$.

\section{Two-stage decoding algorithm}

To reduce the complexity of ML decoding, a decoding algorithm that consists of two decoding stages is proposed. The first decoding stage is responsible for a preliminary decision that serves to roughly find the likely candidates of $\mathcal{B}$ using the QRD-M algorithm. The second decoding stage is responsible for a final decision to recover the message data, which selects the most likely candidate among those determined using the results of the first decoding stage.

\subsection{First decoding stage}

The QRD-M algorithm is a technique that uses QR decomposition combined with the $(M)$-algorithm to reduce the complexity of ML decoding. A block diagram of the transmitter and receiver of $\mathrm{PC} / \mathrm{HC}-\mathrm{MCM}$ discussed in this article is shown in Figure 3.

The QR decomposition of the matrix WF is given by

$$
\mathbf{W F}=\mathbf{Q R},
$$

where $\mathbf{Q}$ is the $M_{t}-$ by $-M_{t}$ unitary matrix $\left(\mathbf{Q}^{H}=\mathbf{Q}^{-1}\right)$

$$
\mathbf{Q}=\left[\begin{array}{ccc}
q_{1,1} & \cdots & q_{1, M_{t}} \\
\vdots & \ddots & \vdots \\
q_{M_{t}, 1} & \cdots & q_{M_{t}, M_{t}}
\end{array}\right]
$$

and $\mathbf{R}$ is the $M_{t}$-by- $M_{c}$ upper triangular matrix

$$
\mathbf{R}=\left[\begin{array}{ccccc}
a_{1,1} & a_{1,2} & \cdots & a_{1, M_{c}-1} & a_{1, M_{c}} \\
0 & a_{2,2} & \cdots & a_{2, M_{c}-1} & a_{2, M_{c}} \\
\vdots & \vdots & \ddots & \vdots & \vdots \\
0 & 0 & \cdots & a_{M_{c}-1, M_{c}-1} & a_{M_{c}-1, M_{c}} \\
0 & 0 & \cdots & 0 & a_{M_{c}, M_{c}} \\
\vdots & \vdots & \ddots & \vdots & \vdots \\
0 & 0 & \cdots & 0 & 0
\end{array}\right] \begin{gathered}
(1) \\
(2) \\
\left(M_{c}-1\right) \\
\left(M_{c}\right) \\
\vdots \\
\left(M_{t}\right)
\end{gathered}
$$

By premultiplying the received signal vector $\hat{\mathbf{y}}$ by the matrix $\mathbf{Q}^{H}$, we obtain

$$
\mathbf{Q}^{H} \hat{\mathbf{y}}=\mathbf{Q}^{H}\left(\mathbf{W F} \mathbf{x}_{\mathcal{B}}+\boldsymbol{\eta}\right)=\mathbf{R} \mathbf{x}_{\mathcal{B}}+\mathbf{Q}^{H} \boldsymbol{\eta} .
$$

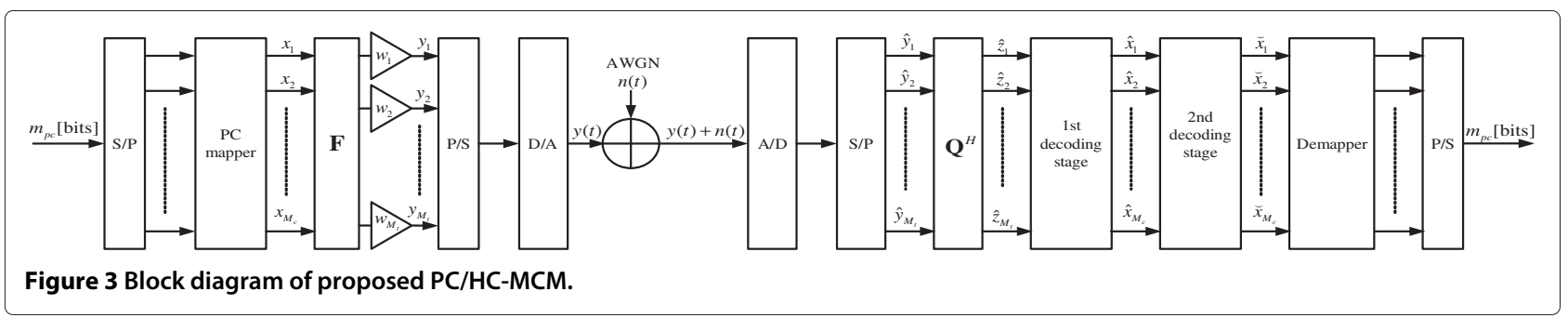




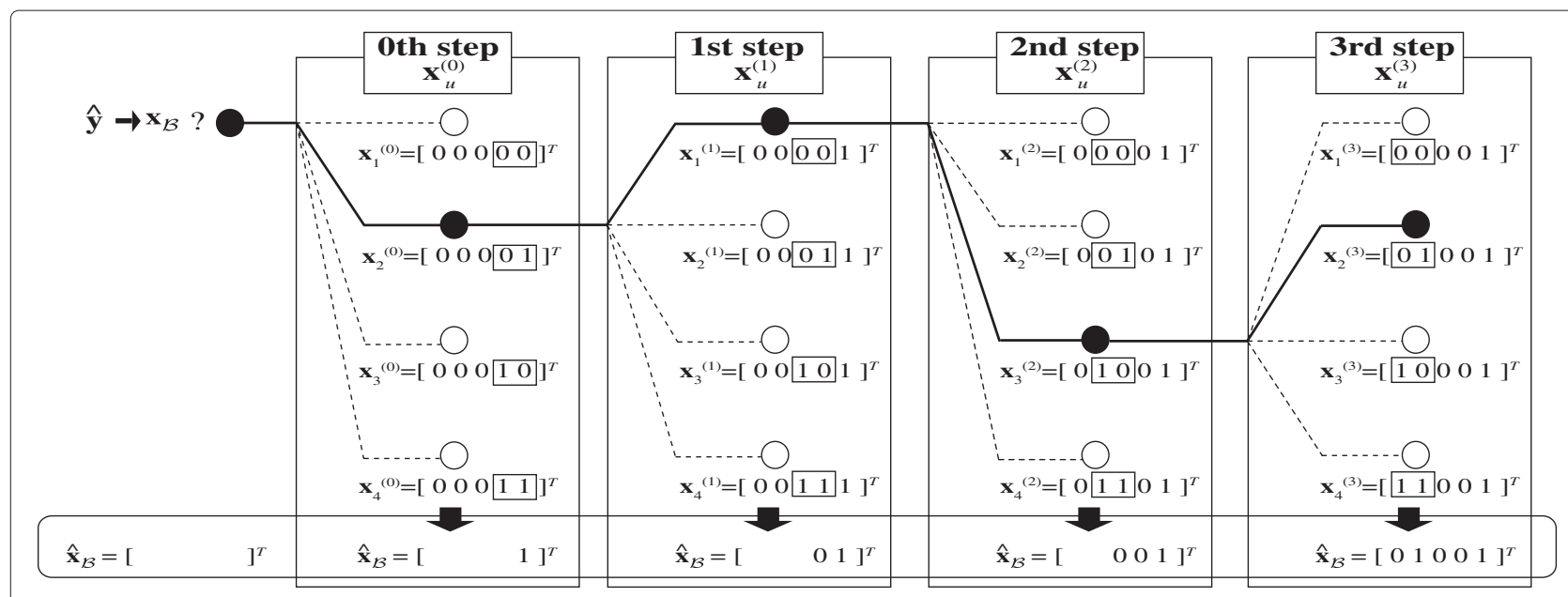

Figure 4 Example of $M$-algorithm decoding $\left(M_{C}=5, M=2\right)$.

Since $\mathbf{Q}^{H} \mathbf{y}=\mathbf{R x}_{\mathcal{B}}$ for the noiseless vector $\mathbf{y}$ of $\hat{\mathbf{y}}$, this formula can be used to produce replica vectors $\mathbf{R} \mathbf{x}_{\mathcal{B}_{i}}$ $\left(\mathcal{B}_{i} \in \mathcal{C}\right)$ such that

$$
\mathbf{R x}_{\mathcal{B}_{i}}=\left[\begin{array}{c}
a_{1,1} x_{i, 1}+\cdots+a_{1, M_{c}} x_{i, M_{c}} \\
\vdots \\
\vdots \\
\vdots \\
a_{M_{c}-1, M_{c}-1} x_{i, M_{c}-1}+a_{M_{c}-1, M_{c}} x_{i, M_{c}} \\
a_{M_{c}, M_{c}} x_{i, M_{c}} \\
0 \\
\vdots \\
0
\end{array}\right] \begin{gathered}
(1) \\
\vdots \\
\left(M_{c}-1\right) \\
\left(M_{c}\right) \\
\vdots \\
\\
\left(M_{t}\right)
\end{gathered}
$$

where $x_{i, l}\left(l=1,2, \ldots, M_{c}\right)$ is the $l$ th element of vector $\mathbf{x}_{\mathcal{B}_{i}}$.
To reduce the number of replica vectors $\mathbf{R} \mathbf{x}_{\mathcal{B}_{i}}$, the proposed method using the $(M)$-algorithm uses vectors $\mathbf{x}_{u}^{(0)}\left(u=1,2, \ldots, U_{0} \leq 2^{M}\right)$ that contain all possible limited $M$ elements of $\mathbf{x}_{\mathcal{B}_{i}}$ at the 0th step ${ }^{\mathrm{a}}$.

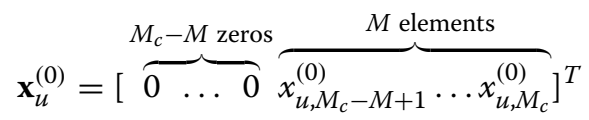

Therefore, using $\mathbf{x}_{u}^{(0)}$, it is expected that a candidate $\hat{x}_{M_{c}}$ of $x_{M_{c}}$ can be efficiently estimated such that

$$
\hat{x}_{M_{c}}=x_{u_{0}, M_{c}}^{(0)}, \quad u_{0}=\arg \min _{u}\left(\left\|\mathbf{Q}^{H} \hat{\mathbf{y}}-\mathbf{R} \mathbf{x}_{u}^{(0)}\right\|\right) .
$$

Vectors $\mathbf{x}_{u}^{(1)}\left(u=1,2, \ldots, U_{1} \leq 2^{M}\right)$ are defined using $\hat{x}_{M_{c}}$ obtained at the 0 th step as

$$
\mathbf{x}_{u}^{(1)}=[\overbrace{0 \ldots 0}^{M_{c}-M-1} \overbrace{x_{u, M_{c}-M}^{(1)} \ldots x_{u, M_{c}-1}^{(1)}}^{M \text { elements }} \hat{x}_{M_{c}}]^{T}
$$

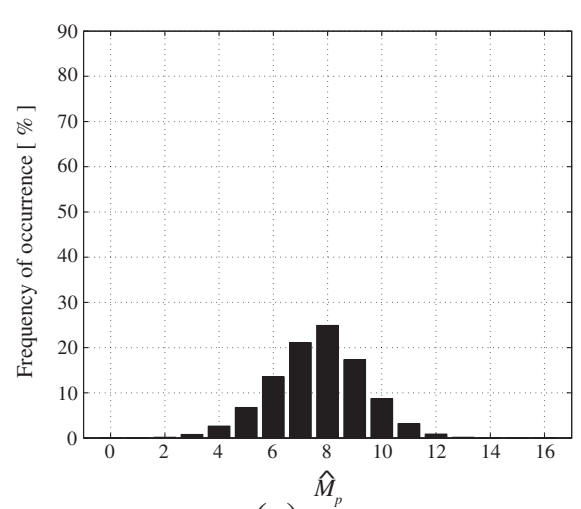

(a) $E_{b}^{p} / N_{0}=0 \mathrm{~dB}$

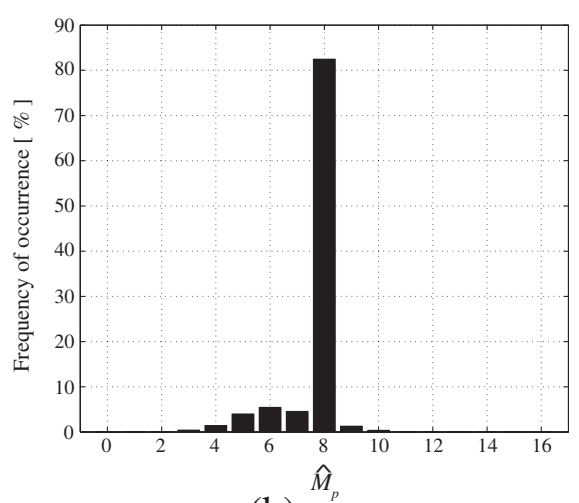

(b) $E_{b}{ }^{\prime} N_{0}=8 \mathrm{~dB}$

Figure 5 Histogram of the Hamming weight of $\hat{\mathbf{x}}_{\mathcal{B}}\left(\left(\begin{array}{c}M_{\boldsymbol{c}} \\ \boldsymbol{M}_{\boldsymbol{p}}\end{array}\right)=\left(\begin{array}{c}\mathbf{1 6} \\ \mathbf{8}\end{array}\right), \boldsymbol{\Delta} \boldsymbol{f} \boldsymbol{T}_{\boldsymbol{s}}=\mathbf{0 . 5}, \boldsymbol{M =} \mathbf{7}\right) \cdot(\mathbf{a}) E_{b} / N_{0}=0 \mathrm{~dB} .(\mathbf{b}) E_{b} / N_{0}=8 \mathrm{~dB}$ 


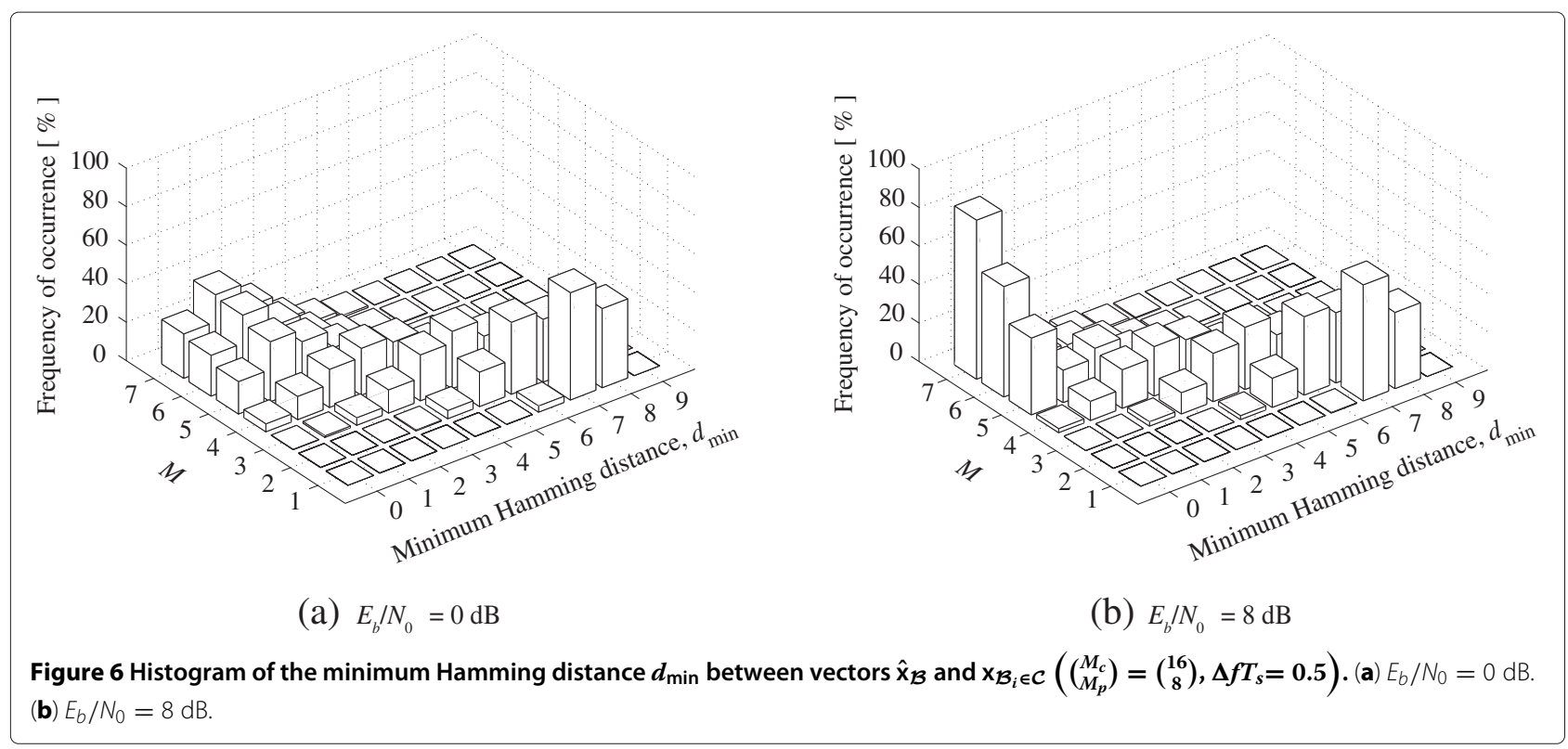

and a candidate $\hat{x}_{M_{c}-1}$ of $x_{M_{c}-1}$ is obtained such that

$$
\hat{x}_{M_{c}-1}=x_{u_{1}, M_{c}-1}^{(1)}, \quad u_{1}=\arg \min _{u}\left(\left\|\mathbf{Q}^{H} \hat{\mathbf{y}}-\mathbf{R} \mathbf{x}_{u}^{(1)}\right\|\right)
$$

at the first step. Similarly, at the $n$th step $(n=$ $\left.0,1, \ldots, M_{c}-M-1\right)$, we obtain $\hat{x}_{M_{c}-n}$ using vectors $\mathbf{x}_{u}^{(n)}\left(u=1,2, \ldots, U_{n} \leq 2^{M}\right)$ given by

$\mathbf{x}_{u}^{(n)}=[\overbrace{0}^{M_{c}-M-n \text { zeros }} \overbrace{x_{u, M_{c}-M-n+1}^{(n)} \ldots x_{u, M_{c}-n}^{(n)}}^{M \text { elements }} \hat{x}_{M_{c}-n+1} \ldots \hat{x}_{M_{c}}]^{T}$,

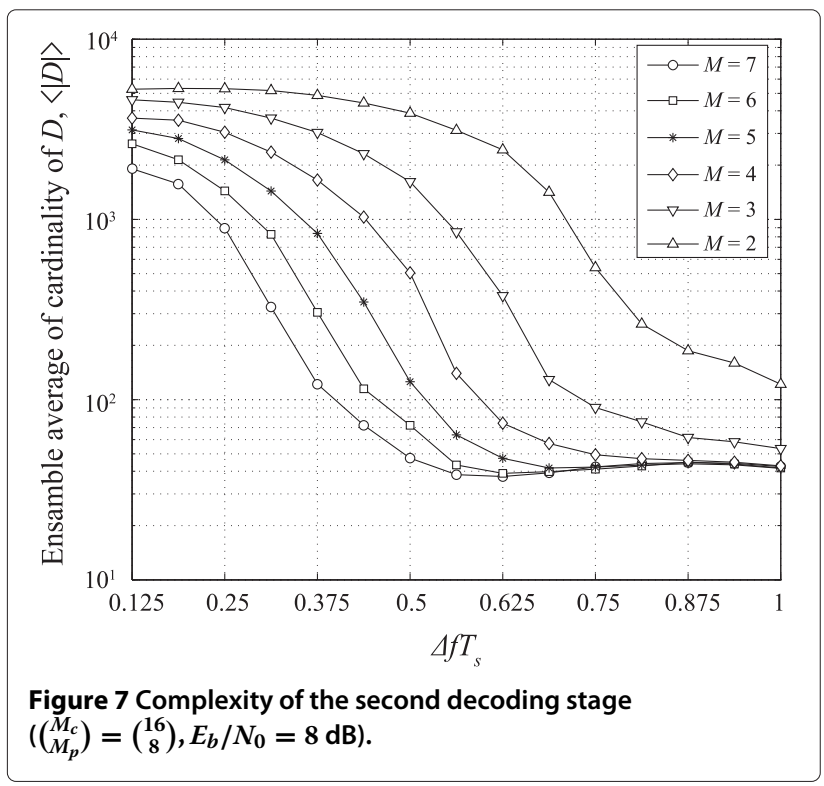

such that

$$
\hat{x}_{M_{c}-n}=x_{u_{n}, M_{c}-n}^{(n)}, \quad u_{n}=\arg \min _{u}\left(\left\|\mathbf{Q}^{H} \hat{\mathbf{y}}-\mathbf{R} \mathbf{x}_{u}^{(n)}\right\|\right) .
$$

At the $\left(M_{c}-M\right)$ th step, we simultaneously obtain the candidates $\hat{x}_{1}, \ldots, \hat{x}_{M}$. Therefore, $\hat{x}_{1}$ is obtained at the $\left(M_{c}-M\right)$ th step, and, after performing all of the above steps, we finally have $\hat{\mathbf{x}}_{\mathcal{B}}=\left[\begin{array}{llll}\hat{x}_{1} & \hat{x}_{2} & \ldots & \hat{x}_{M_{c}}\end{array}\right]^{T}$.

An example of the $(M)$-algorithm for $M_{c}=5$ and $M=$ 2 is illustrated in Figure 4. Note that the computational complexity for the first decoding stage is deterministic if the parameter $M$ is fixed at the receiver.

\subsection{Second decoding stage}

The primary purpose of the second decoding stage shown in Figure 3 is to solve the problem that the number of nonzero elements (ones), $\hat{M}_{p}$ (the Hamming weight), contained in the vector $\hat{\mathbf{x}}_{\mathcal{B}}$ is not necessarily equal to the integer $M_{p}$ adopted for PC signaling.

Table 2 Specifications of the simulations

\begin{tabular}{cc}
\hline System item & Parameter \\
\hline$\left(\begin{array}{c}M_{c} \\
M_{p}\end{array}\right), m_{p c}$ & $\left(\begin{array}{c}16 \\
8\end{array}\right), 13$ \\
$M$ & $2,3,4,5,6,7$ \\
$M_{t}$ & 32 \\
Window & Half-wave sinusoid \\
$P C$ mapping & Gray code mapping \\
Noise & AWGN \\
Synchronization & Complete \\
\hline
\end{tabular}


Figure 5 shows examples of histograms obtained through simulations for $\left(\begin{array}{l}M_{c} \\ M_{p}\end{array}\right)=\left(\begin{array}{c}16 \\ 8\end{array}\right), \Delta f T_{s}=0.5$, and $M=7$. In Figure $5 \mathrm{a}, E_{b} / N_{0}$ was chosen to be $0 \mathrm{~dB}$, and in Figure $5 \mathrm{~b}, E_{b} / N_{0}$ was chosen to be $8 \mathrm{~dB}$, where $E_{b}$ is the average energy per bit of the PC/HC-MCM signals. Although the most frequently occurring value of $\hat{M}_{p}$ is 8 (= $M_{p}$ ), the probability that $\hat{M}_{p}$ takes different integers is not negligible. This means that the vector $\hat{\mathbf{x}}_{\mathcal{B}}$ obtained at the first decoding stage is not necessarily included in the possible set of vectors $\mathbf{x}_{\mathcal{B}_{i} \in \mathcal{C}}$, which directly results in an increase in the BER. Therefore, we use the second decoding stage to reduce the error contained in $\hat{\mathbf{x}}_{\mathcal{B}}$. In the second decoding stage, we use the Hamming distances $d_{H}\left(\hat{\mathbf{x}}_{\mathcal{B}}, \mathbf{x}_{\mathcal{B}_{i} \in \mathcal{C}}\right)$ between vectors $\hat{\mathbf{x}}_{\mathcal{B}}$ and $\mathbf{x}_{\mathcal{B}_{i} \in \mathcal{C}}$ to search for a subset $\mathcal{D} \subset \mathcal{C}$, which corresponds to the set of vectors $\mathbf{x}_{\mathcal{B}_{i} \in \mathcal{D}}$ that have the minimum value $d_{\text {min }}$ of the Hamming distances $d_{H}\left(\hat{\mathbf{x}}_{\mathcal{B}}, \mathbf{x}_{\mathcal{B}_{i} \in \mathcal{C}}\right)$. In the second decoding stage, all the vectors $\mathbf{x}_{\mathcal{B}_{i} \in \mathcal{C}}$ are compared with $\hat{\mathbf{x}}_{\mathcal{B}}$ to obtain the subset $\mathcal{D}$. This involves logical computations, which require fewer computations than the multiplications involved in the Euclidean distance calculations.

Figure 6 shows histograms of the minimum Hamming distance $d_{\min }$ for various values of the parameter $M$ in the $(M)$-algorithm. In Figure $6 \mathrm{a}, E_{b} / N_{0}$ was chosen to be $0 \mathrm{~dB}$, and in Figure $6 \mathrm{~b}, E_{b} / N_{0}$ was chosen to be $8 \mathrm{~dB}$. Figure $6 \mathrm{a}, \mathrm{b}$ show that the minimum Hamming distance $d_{\text {min }}$ depends greatly on the values of $M$ and $E_{b} / N_{0}$. However, for high $E_{b} / N_{0}$ and $M \geq 5, d_{\text {min }}=0$ occurs with higher probability. In such a case, the computational complexity is not strongly affected by the second decoding stage, because the second decoding stage does not work for $d_{\text {min }}=0$. However, the computational complexity of the first decoding stage increases with the value of $M$.

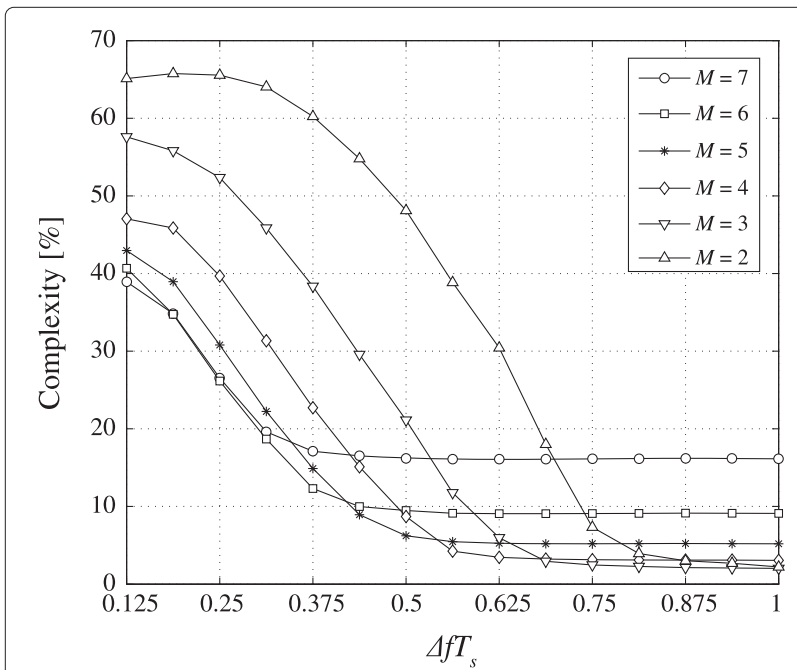

Figure 8 Total complexity of the two-stage decoding algorithm $\left(\left(\begin{array}{c}M_{c} \\ M_{p}\end{array}\right)=\left(\begin{array}{c}16 \\ 8\end{array}\right), E_{b} / N_{0}=8 \mathrm{~dB}\right)$.
Using the subset $\mathcal{D}$, the second decoding stage decodes the message data such that

$$
\check{\mathbf{x}}_{\mathcal{B}}=\arg \min _{\mathcal{B}_{i} \in \mathcal{D}}\left(\left\|\mathbf{Q}^{H} \hat{\mathbf{y}}-\mathbf{R} \mathbf{x}_{\mathcal{B}_{i}}\right\|\right)
$$

\section{Results}

\subsection{Computational complexity}

As mentioned in Section 3.1, the computational complexity of the first decoding stage is deterministic, whereas that of the second decoding stage is probabilistic. Therefore, we first discuss the computational complexity for the second decoding stage. To evaluate the complexity of the second decoding stage, we perform a simulation to obtain the ensemble average of the cardinality of the subset $\mathcal{D},\langle|\mathcal{D}|\rangle$, which corresponds to the average number of Euclidean distance calculations required to solve the problem given by (26). The results are shown in Figure 7, where $\left(\begin{array}{l}M_{c} \\ M_{p}\end{array}\right)$ and $E_{b} / N_{0}$ are chosen to be $\left(\begin{array}{c}16 \\ 8\end{array}\right)$ and $8 \mathrm{~dB}$, respectively. Other specifications of the simulations can be seen in Table 2. Figure 7 shows that $\langle|\mathcal{D}|\rangle$ decreases with increasing $\Delta f T_{s}$ and $M$, and that $\langle|\mathcal{D}|\rangle$ is independent of the value of $\Delta f T_{s}$ if $\Delta f T_{s} \gtrsim 0.5$ for $M \geq 5$, where the curves are slightly nonmonotonic.

Figure 8 shows the total complexity of the proposed two-stage decoding algorithm relative to the complexity of $\mathrm{ML}$ decoding as a function of $\Delta f T_{s}$. The complexity is defined by the total number of calculations of the Euclidean distance required to obtain $\hat{\mathbf{x}}_{\mathcal{B}}$ and $\check{\mathbf{x}}_{\mathcal{B}}$. Figure 8 shows that for larger values of $\Delta f T_{s}$, the complexity remains constant ${ }^{\mathrm{b}}$ and takes a smaller value. For example, the complexity can be reduced to 3 to $6 \%$ of the complexity of ML decoding when $\Delta f T_{s} \gtrsim 0.5$ and $M=4$ or 5 .

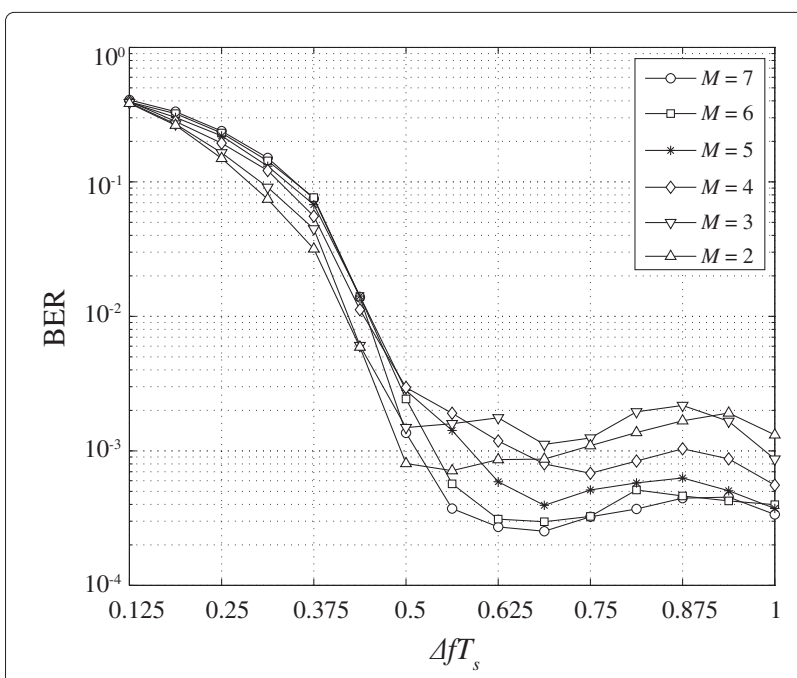

Figure 9 BER versus $\Delta f T_{s}$ for PC/HC-MCM using the two-stage decoding algorithm $\left(\left(\begin{array}{c}M_{c} s \\ M_{p}\end{array}\right)=\left(\begin{array}{c}16 \\ 8\end{array}\right), E_{b} / N_{0}=8 \mathrm{~dB}\right)$. 


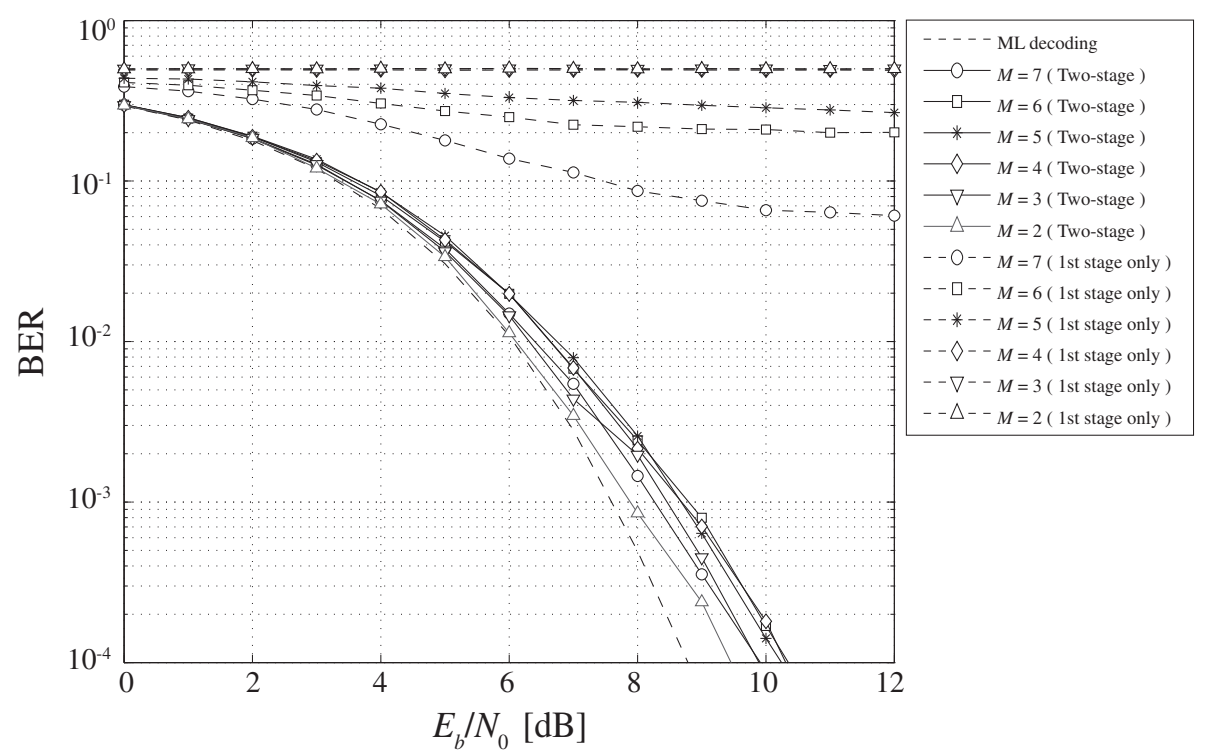

Figure 10 BER performance of PC/HC-MCM using the two-stage decoding algorithm $\left(\left(\begin{array}{c}M_{c} \\ M_{p}\end{array}\right)=\left(\begin{array}{c}16 \\ 8\end{array}\right), \Delta f T_{s}=0.5\right)$.

\subsection{BER performance}

The BER versus $\Delta f T_{s}$ characteristics for $\mathrm{PC} / \mathrm{HC}-\mathrm{MCM}$ using the proposed two-stage decoding algorithm are shown in Figure 9 for $E_{b} / N_{0}=8 \mathrm{~dB}$. It can be observed that the BER curves in Figure 9 exhibit some fluctuation when $\Delta f T_{s} \geq 0.5$. This is caused by intercarrier interference (ICI), which depends on the value of $\Delta f T_{s}$. Figure 10 shows the BER versus $E_{b} / N_{0}$ characteristics, where solid lines denote the results of the proposed decoding algorithm and dashed lines denote the results of ML decoding with the first decoding stage only. Figure 10 shows that the BER characteristics of PC/HC-MCM using the proposed decoding algorithm have a loss of less than approximately $1.5 \mathrm{~dB}$ in $E_{b} / N_{0}$ at a BER of $10^{-3}$ as compared with that of $\mathrm{PC} / \mathrm{HC}-\mathrm{MCM}$ using $\mathrm{ML}$ decoding. However, as shown in Figure 8, the computational complexity is greatly reduced by the proposed algorithm. It can be seen from Figure 10 that the best performance of the twostage decoding algorithm is obtained with $M=2$, for which the second decoding stage is dominant and the total computational complexity is the highest, even though the computational complexity of the first decoding stage using the $(M)$-algorithm is the lowest.

\section{Conclusion}

A decoding algorithm that consists of two decoding stages to reduce the complexity of decoding for PC/HC-MCM signals was proposed. The proposed two-stage decoding algorithm was demonstrated to be effective for reducing the computational complexity with only a small increase in BER. According to results obtained considering the equalization at the receiver [11], we believe that our proposed two-stage decoding algorithm can also be used in intersymbol interference (ISI) channels. In the near future, we will take the multipath and other ISI channels into consideration to develop a lower-complexity decoding algorithm for PC/HC-MCM.

\section{Endnotes}

${ }^{\text {a } I n}$ the ordinary $M$-algorithm, the parameter $M$ is used to limit maximum number of surviving branches. In this article, we only choose a single surviving branch to reduce the complexity, and $M$ is the number of bits considered at each step. Thus, the meaning of the parameter $M$ is different from that in the ordinary $M$-algorithm.

${ }^{\mathrm{b}}$ As was shown in Figure 7 , the complexity curves of $\langle|\mathcal{D}|\rangle$ for $\Delta f T_{s} \geq 0.5$ and $M=4$ or 5 are nonmonotonic. However, such nonmonotonic behavior cannot be seen in Figure 8 because in the nonmonotonic parts in Figure 8, the complexity of the first decoding stage is dominant and the nonmonotonic behavior cannot be distinguished.

\section{Competing interests}

The authors declare that they have no competing interests.

Received: 20 August 2012 Accepted: 5 February 2013

Published: 16 March 2013

\section{References}

1. M Itami, Fundamentals of OFDM and its application. Fundament. Rev. $\mathbf{1}(2), 35-43$ (2007)

2. RV Nee, R Prasad, OFDM for Wireless Multimedia Communications. (Artech House, Boston, 2000)

3. L Hanzo, M Munster, BJ Choi, T Keller, OFDM and MC-CDMA for Broadband Multi-user Communications, WLANs and Broadcasting. (Wiley, West Sussex, 2003) 
4. J Zhu, S Sasaki, G Marubayashi, Proposal of parallel combinatory spread spectrum communication system. IEICE Trans. Commun. J74-B(5), 207-214 (1991)

5. S Sasaki, H Kikuchi, J Zhu, G Marubayashi, Multiple access performance of parallel combinatory spread spectrum communication systems in nonfading and Rayleigh fading channels. IEICE Trans. Commun. E78-B(8), 1152-1161 (1991)

6. SY Park, SB Yun, CG Kang, Performance of multi-carrier parallel combinatory DS-CDMA system. IEICE Trans. Commun. E81-B(9), 1758-1769 (1998)

7. KTakizawa, S Sasaki, S Muramatsu, J Zhou, H Kikuchi, Performance bounds in convolutional coded parallel combinatorial SS systems. IEICE Trans. Fundament. E85-A(7), 1696-1701 (2002)

8. K Ohuchi, Constant amplitude signaling for parallel combinatory spread spectrum systems. IEICE Trans. Fundament. E89-A(9), 2329-2336 (2006)

9. PK Frenger, N Arne, B Svensson, Parallel combinatory OFDM signaling. IEEE Trans. Commun. 47(4), 558-565 ( 1999)

10. M Hamamura, S Tachikawa, in Proceedings of the 15th IEEE International Symposium on Personal, Indoor and Mobile Radio Communications, vol. 1. Bandwidth efficiency improvement for multi-carrier systems, (IEEE, Barcelona, 2004), pp. 48-52

11. Y Hou, M Hamamura, A novel modulation with parallel combinatory and high compaction multi-carrier modulation. IEICE Trans. Fundament. E90-A(11), 2556-2567 (2007)

12. G Ungerboeck, Adaptive maximum-likelihood receiver for carrier-modulated data-transmission systems. IEEE Trans. Commun. 20(5), 624-636 (1974)

13. STakahashi, M Hamamura, S Tachikawa, in Proceedings of the 1st International Symposium on Wireless Communications Systems, vol. 1. A demodulation complexity reduction method using M-algorithm for high compaction multicarrier modulation systems (University of Bristol, Mauritius, 2004), pp. 418-422

14. Y Morishige, M Fujii, M Itami, K Itoh, Tree search detection based on LLR Using M algorithm in MC-CDMA systems. IEICE Trans. Fundament. E89-A(10), 2622-2629 (2006)

15. K Adachi, M Nakagawa, Iterative modified QRD-M based on CRC codes for OFDM MIMO multiplexing. IEICE Trans. Commun. E90-B(6), 1433-1443 (2007)

16. KJ Kim, Jltis Yue R A, JD Gibson, A QRD-M/Kalman filter-based detection and channel estimation algorithm for MIMO-OFDM systems. IEEE Trans. Wirel. Commun. 4(2), 710-721 (2005)

17. JB Anderson, S Mohan, Sequential coding algorithms: a survey and cost analysis. IEEE Trans. Commun. 32(2), 169-176 (1984)

18. R Hayashi, M Hamamura, in Proceedings of the 5th International Conference on Innovations in Information Technology, vol. 1. Complexity-reduced decoding algorithm for unmodulated parallel-combinatory high-compaction multicarrier modulation signals (IEEE, Al Ain, 2008), pp. 49-53

19. AH Nuttall, $F$ Amoroso, Minimum Gabor bandwidth of $M$, orthogonal signals. IEEE Trans. Inf. Theory. 11(3), 440-444 (1965)

doi:10.1186/1687-1499-2013-75

Cite this article as: Hayashi et al:: Two-stage decoding algorithm for unmodulated parallel combinatory high-compaction multicarrier modulation signals. EURASIP Journal on Wireless Communications and Networking 2013 2013:75.

\section{Submit your manuscript to a SpringerOpen ${ }^{\odot}$ journal and benefit from:}

- Convenient online submission

- Rigorous peer review

- Immediate publication on acceptance

- Open access: articles freely available online

- High visibility within the field

- Retaining the copyright to your article 